DE

M E D I C I N A

T R O P I C A L

$\mathrm{DE}$

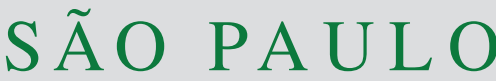

JOURNAL OF THE SÃO PAULO INSTITUTE OF TROPICAL MEDICINE

1 Universidade de São Paulo, Instituto de Medicina Tropical de São Paulo, Laboratório de Soroepidemiologia e Imunobiologia, São Paulo, São Paulo, Brazil

2Universidade de São Paulo, Faculdade de Medicina, Hospital das Clínicas, Instituto da Criança e do Adolescente, Unidade de Vacinas e Imunobiológicos Especiais, São Paulo, São Paulo, Brazil

${ }^{3}$ Universidade de São Paulo, Faculdade de Medicina, Hospital das Clínicas, Laboratório de Investigação Médica (LIM-48), São Paulo, São Paulo, Brazil

${ }^{4}$ Universidade de São Paulo, Faculdade de Medicina, Departamento de Pediatria, São Paulo, São Paulo, Brazil

Correspondence to: Kelly Aparecida Kanunfre

Universidade de São Paulo, Instituto de Medicina Tropical de São Paulo, Laboratório de Soroepidemiologia e Imunobiologia, Av. Dr Eneas de Carvalho Aguiar, 470, CEP 05403-000, São Paulo, SP, Brazil Tel: +55 1130617022

E-mail: kanunfre@usp.br

Received: 21 July 2020

Accepted: 21 August 2020

\section{SARS-CoV-2 infections with emphasis on pediatric patients: a narrative review}

\author{
Lidia Yamamoto', Emilly Henrique dos Santos ${ }^{\circledR 1}$, Lacyane Santos Pinto', \\ Mussya Cisotto Rocha ${ }^{1,3}$, Kelly Aparecida Kanunfre ${ }^{\circledR 1,3}$, Marcelo Genofre \\ Vallada $^{2}$, Thelma Suely Okay ${ }^{\circledR 1,4}$
}

\section{ABSTRACT}

This narrative review summarizes the main aspects underlying the new coronavirus SARS-CoV-2, its epidemiology, pathophysiology, pointing to differences of SARS-CoV-2 main receptors ACE2, in terms of expression and the amount of soluble ACE2 in the circulation of children, men and women, and also in those with risk factors such as the smokers and pregnant women or presenting with comorbidities (diabetes, obesity, hypertension and other cardiovascular diseases, renal and CNS pre-existing diseases). Clinical manifestations in adults and children were also described, emphasizing the particularities already seen in children, regarding signs, symptoms, viral excretion time and the involvement of all organs and systems. The COVID-19 in the pediatric population was divided into two sections: one dedicated to previously healthy children and adolescents with COVID-19, and the other to those who live with comorbidities and acquired COVID-19. A few paragraphs were reserved to the recently described severe multisystemic inflammatory syndrome associated with COVID-19 (MIS-C) that shares certain characteristics with Kawasaki disease. Some studies on the infection in pregnant and postpartum women, as well as neonates were shown. This review has also covered the laboratory diagnosis of COVID-19, passing through the imaging diagnosis made by the chest tomography revealing ground glass patching opacities, and results of non-specific exams such as the total blood with lymphopenia, the coagulation tests with increased prothrombin times, as well as marked increments of the D-dimer, troponin and proinflammatory cytokines. In the section devoted to the specific laboratory diagnosis of COVID-19, the most used RT-PCR protocols were described and some studies on the serological diagnosis with $\operatorname{IgA}$, IgM and IgG detection were detailed, including the use of rapid immunochromatographic assays and discussing the ideal period after the onset of symptoms to perform each type of test. In the end, the management of pediatric patients with COVID-19 based mainly on supportive measures has been briefly commented.

KEYWORDS: COVID-19. SARS-CoV-2. Pediatric COVID-19. SARS-CoV-2 in children and adolescents. Maternal COVID-19. Fetal and neonatal COVID-19.

\section{INTRODUCTION}

In December 2019, an outbreak of pneumonia of unknown etiology was reported in the province of Hubei, city of Wuhan, China. In the following month, the etiologic agent was isolated and had its genome sequenced, revealing a new coronavirus, the SARS-CoV-2 or 2019-nCoV, and the disease was called COVID-19. The origin of the outbreak was attributed by the Chinese government to a local seafood market. 
On March, 11, 2020, the World Health Organization (WHO) declared COVID-19 a pandemic ${ }^{1}$ and on July 20, 2020, there are more than 14 million cases and 605 thousand deaths by COVID-19 around the world, and in Brazil the number of confirmed cases has surpassed 2 million with more than 80 thousand deaths.

\section{Etiology}

SARS-CoV-2 is one of seven coronaviruses that infect humans identified to date. The strains 229E, NL63, OC43 and HKU1 cause mild infections of the upper respiratory tract in all age groups, while SARS-CoV (severe acute respiratory syndrome coronavirus) and MERS-CoV (Middle East respiratory syndrome coronavirus), both of zoonotic origin, cause severe respiratory failure, with $10 \%$ and $35 \%$ of mortality rates, respectively ${ }^{2}$.

SARS-CoV-2 is a round-shaped enveloped coronavirus with a diameter between 60-140 nanometers, containing a positive sense single-stranded RNA genome (ssRNA +). It belongs to the $\mathrm{B}$-line of $\beta$-coronavirus, subgenus Sarbecovirus, identified in humans, other mammals and birds. The SARS-CoV-2 genome shares a similarity of $79.5 \%$ with SARS-CoV, $50 \%$ with MERS-CoV and $96 \%$ with the bat coronavirus RaTG133.

The complete SARS-CoV-2 genome is about $30 \mathrm{~kb}$ in length. The 5' end contains two thirds of the viral genome, corresponding to the Open Reading Frame ORF1ab which produces two viral replicases, the polyproteins PP1a and $\mathrm{PP} 1 \mathrm{ab}$, that, in turn, after processing, give rise to 16 mature non-structural proteins (nsp) forming the replicasetranscriptase complex. The 3' end of the viral genome encodes four structural proteins: spike (S), envelope (E), membrane $(\mathrm{M})$ and nucleocapsid $(\mathrm{N})$, in addition to accessory genes ${ }^{3}$.

In SARS-CoV-2, the gene encoding the S protein shares only $75 \%$ of amino acid identity with its counterpart gene in SARS-CoV. The other three structural proteins (E, M and N) are more conserved and involved in the RNA encapsulation, in the envelope and other proteins formation, as well as in budding ${ }^{3}$.

The complete genome analysis of 257 SARS-CoV-2 isolates identified at least five viral clades with great genetic diversity, but without clusters formation according to geographic areas. The 5' end of the virus is more susceptible to mutations than the 3 ' one, and in the latter, the most susceptible regions to mutations are the ORF3a, E gene, $S$ gene with special emphasis on the RBD (Receptor Binding Domain) of the viral spike encoded by the $S$ gene which is a mutational hotspot ${ }^{4}$.

\section{Epidemiology}

The incubation period of COVID-19 ranges from 3-14 days, with a basic reproduction number (R0) varying from 1.4 to 6.49 , depending on the country and the adopted control measures ${ }^{5}$.

SARS-CoV-2 is transmitted by droplets released by sneezing and coughing. Asymptomatic individuals might transmit the infection, as some reports described individuals in whom high viral loads were detected in respiratory secretions during the early stages of the infection, even before the onset of symptoms ${ }^{5}$.

According to the severity of clinical manifestations, COVID-19 can be classified as asymptomatic or symptomatic, the latter ranging from mild to severe and critical. Mortality due to COVID-19 was $1.4 \%$ in China and up to $15.2 \%$ in other countries ${ }^{6}$.

COVID-19 affects most frequently people over 60 years of age, especially smokers and those with comorbidities such as diabetes, hypertension, cardiovascular disease, obesity, chronic lung disease, kidney diseases ${ }^{5}$.

What has drawn attention since the beginning of the pandemic is the low number of children and adolescents with severe and critical COVID-19, and the large number of asymptomatic infections in this age group ${ }^{7}$.

\section{Pathophysiology and clinical presentation}

SARS-CoV and SARS-CoV-2 have high affinity with the angiotensin-converting enzyme 2 cellular transmembrane receptors (ACE2), and this affinity is 10 to 20 times higher to SARS-CoV-2. The viral binding with ACE2 receptors is the main mechanism of viral entry into the epithelial cells mainly of the lungs (type II pneumocytes), intestines (enterocytes), kidneys and blood vessels. This entry takes place through the binding of the viral protein $\mathrm{S}$ (spike) receptor, more specifically of the receptor binding domain (RBD) region with the host cell transmembrane ACE2 receptor. Then, after the intronization of SARS-CoV-2 and the ACE2 receptor, the $\mathrm{S}$ protein is cleaved by the transmembrane serine protease TMPRSS2 and the viral RNA is released into the host cell, being ready to initiate the viral replication. The ACE2 receptor, in turn, will undergo cleavage by TACE (tumor necrosis factor alpha-converting enzyme), also known as ADAM 17, which is a metalloprotease that allows the shedding of the ACE2 ectodomain, known as soluble or circulating ACE2, into the extracellular space. Soluble ACE2 is enzymatically active and apparently able to bind with SARS-CoV-2, supporting the rationale for the administration of recombinant human ACE2 to reduce the deleterious inflammatory effects of SARS-CoV-2 infections ${ }^{8}$. 
COVID-19 symptomatology is nonspecific and includes fever, dry cough, dyspnea, headache, myalgia, fatigue, nausea, vomiting and abdominal pain ${ }^{5}$. In children, COVID-19 symptoms do not differ from those of adults, although a higher proportion of asymptomatic and oligosymptomatic patients has been reported. In addition, there is a more prolonged viral excretion in respiratory secretions for up to 22 days after the onset of symptoms and in feces for more than 30 days $^{9}$.

Cardiac complications associated with COVID-19 have been frequently reported in adult patients and some of them can occur in severely ill children. The pathophysiology of cardiovascular complications involves the direct viral injury, hypoxemia, hemodynamic instability leading to hypoperfusion, enhanced systemic inflammation, local regulation of the expression of ACE2 receptors, enhanced production of catecholamines and a higher incidence of medication toxicity. These signs and symptoms lead to chest pain due to acute cardiac injury (8-12\%), heart failure (23-52\%), arrhythmia (8.9-16.7\%), acute myocarditis and ultimately shock ${ }^{10}$.

SARS-CoV-2 can invade endothelial cells and enterocytes of the small intestine, being detected in fecal samples, esophagus, stomach, duodenal and rectal samples. Patients present with anorexia (26.8\%), diarrhea (12.5\%), nausea and vomiting $(10.2 \%)$, abdominal pain $(9.2 \%)^{11}$.

Acute kidney injury due to SARS-CoV-2 infections occur in $0.5 \%$ of the patients overall, and in up to $23 \%$ of the severe cases that may progress to renal failure, sometimes requiring hemodialysis ${ }^{12}$.

ACE2 receptors are also present in the nervous system and in skeletal muscles, suggesting a mechanism for SARS-CoV-2-related neuromuscular injuries. Dizziness $(16.8 \%)$, headache $(13.1 \%)$, skeletal muscle injury $(10.7 \%)$ are more frequently seen, but COVID-19 can also lead to impaired consciousness (7.5\%), acute cerebrovascular disease (2.8\%), ataxia $(0.5 \%)$, seizures $(0.5 \%)$, meningoencephalitis and the Guillain-Barré syndrome ${ }^{13}$.

Olfactory and gustatory disorders have been associated with viral infections and SARS-CoV-2 is not an exception. Hyposmia (5.1-20.4\%), anosmia (79.6\%), dysgeusia (8.5\%) and ageusia (1.7\%) are the most common symptoms. A recent study has shown that ACE2 is also highly expressed on the oral mucosa, granting the virus an easy access to the host ${ }^{14}$. This finding may explain, at least partially, the dysgeusia and ageusia reported in COVID-19. On the contrary, a reduced expression and activity of ACE2 receptors on the oral, nasal and respiratory epithelium of children has been reported and could explain the lower proportion of serious lung injuries of COVID-19 in children ${ }^{15}$.
Although ACE2 receptors have been detected in the retina, choroid and conjunctival epithelia, ocular involvement associated with SARS-CoV-2 infection, mainly acute conjunctivitis, has been reported in $31.6 \%$ of the patients ${ }^{16}$.

Skin lesions are unfrequently observed in COVID-19, are highly polymorphic and have been reported in some pediatric patients presenting with chilblain-like skin lesions, urticarial eruptions, diffuse or disseminated erythema multiforme-like lesions, livedo racemosa, livedo reticularis, blue toe syndrome, retiform purpura, purpuric, non- purpuric or flexural exanthema, vesicles, acro-ischemic lesions ${ }^{17}$.

Another interesting feature of COVID-19, observed so far in adult patients, is the higher proportion of deaths in men with respect to women. Estrogens seem to participate in the upregulation of ACE2 receptors activity leading to higher amounts of circulating ACE2 in women. A recent report has shown that inhibitors of the 5 alpha reductases, a class of drugs commonly prescribed for prostatic disorders, dampen the androgen signaling and can reduce the expression of ACE2 receptors and of the transmembrane serine protease TMPRSS2, theoretically leading to a decrement in SARS-CoV-2 binding with ACE2 receptors ${ }^{18}$.

Long-term cigarette smokers and obese people have reduced circulating ACE2 levels. In diabetics, the circulating ACE2 down regulates the release of insulin by pancreatic beta cells worsening the glucose control. In pregnant women, the fluctuation of hormones, mainly of progesterone, may drastically upregulate the expression of ACE2 receptors in the uterus and in other organs such as the kidneys, suggesting that pregnant women with COVID-19 could be at higher risk to develop more severe infections ${ }^{19}$.

In summary, it is possible that higher levels of ACE2 in the circulation binding with SARS-CoV-2 could reduce the number of viruses able to bind with ACE2 receptors. A trend toward this same kind of association has been proposed in children, who generally have higher levels of circulating ACE2. In children from 6 months to 17 years of age, ACE2 levels are 13-100 U/liter in comparison with 9-67 U/liter in adults, according to enzyme activity tests ${ }^{20}$.

\section{COVID-19 in the pediatric population}

Zimmermann and Curtis ${ }^{21}$ claimed that children have the same chance of contracting COVID-19 than adults, but they develop more intestinal symptoms and less severe conditions. Children with COVID-19 generally have contact with an infected family member, while the exposure of adults usually occurs in hospitals. 
Ong et $a .^{22}$ called attention to the group of children under one year of age, in whom the occurrence of severe cases of COVID-19 is more frequent than in older children. Lymphopenia, so common in adults $(70 \%)$ occurs in only $3.5 \%$ of children, and the increment of inflammatory markers and cytokines are not so marked, except in the multisystem inflammatory syndrome associated with COVID-19 (MIS-C) cases.

Liguoro et al. ${ }^{23}$ carried out a systematic review on children and adolescents with COVID-19 (0-18 years), through the analysis of 65 articles published from February to May 2020 , totaling 7,480 children, $52.1 \%$ of boys with a mean age of 7.6 years. Most infections were mild $(42.5 \%)$ or moderate $(39.6 \%)$, only $2 \%$ needed ICU and the mortality rate was $0.08 \%$. However, the proportion of newborns with severe infections was $12 \%$; $40 \%$ of them developed dyspnea, while in the other age groups the most frequent symptoms were fever (51.6\%) and cough (47.3\%). Laboratory tests did not show significant changes; $73.9 \%$ of the patients underwent chest tomography and $67.3 \%$ of these exams revealed abnormalities.

Lu et al. ${ }^{24}$ described 1,391 children in Wuhan with severe acute respiratory syndrome. Of these, 171 (12.3\%) had COVID-19, the mean age was 6.7 years, $60.8 \%$ were boys, $41.5 \%$ had fever, $48.5 \%$ cough, $64.9 \%$ pneumonia, three children needed ICU $(0.2 \%)$ and one child died $(0.07 \%)$.

A multicenter cohort study involving 82 healthcare institutions across 25 European countries included 582 children with PCR-confirmed SARS-CoV-2 infection, $62 \%$ of whom were inpatients and $8 \%$ admitted to the ICU. The median age was 5 years, and $87 \%$ of children did not require respiratory support at any time, while $4 \%$ received mechanical ventilation for a median of seven days. The factors associated with ICU admissions were being less than 30 days of life, male sex, preexisting comorbidities and lower respiratory tract symptoms on admission. Four children, all of them older than 10 years, $\operatorname{died}^{25}$.

Jiang et al. ${ }^{26}$ described 161 children with severe acute respiratory syndrome hospitalized in Wuhan, from whom 239 respiratory viruses were identified with predominance of the respiratory syncytial virus in 76 children $(31.80 \%)$ and influenza $\mathrm{A}$ in $72(30.13 \%)$. SARS-CoV-2 was confirmed in only two cases by RT-PCR $(0.84 \%)$, both children were coinfected by respiratory syncytial virus and human metapneumovirus or human metapneumovirus and metapneumovirus. The identification of coinfections could explain the severity of respiratory symptoms in these children.

Recent data reported a severe multisystem inflammatory syndrome in children (MIS-C), adolescents and young adults with respiratory symptoms and positive RT-PCR to SARS-CoV-2, or even without respiratory symptoms, but with positive SARS-CoV-2 serology. Clinical signs and symptoms are highly variable and include some characteristics of the Kawasaki disease, with a tendency to thrombus formation and progression to shock. There is persistent fever, high levels of inflammatory markers such as C-Reactive Protein, troponin and cytokines. More than $50 \%$ of these patients present with cutaneous rash, abdominal pain, nausea, vomiting and diarrhea, and curiously, less than $50 \%$ of them have respiratory symptoms. Some patients needed ICU to receive cardiac (blood pressure maintenance) and respiratory support (Pediatric Intensive Care Society, 2020). Whether this multisystem inflammatory syndrome (MIS-C) associated with COVID-19 will increase in incidence overtime, it is probably dependent on the host genetic susceptibility and/ or the pathogenicity of SARS-CoV-2 genetic variants ${ }^{27,28}$.

Feldstein et $a{ }^{27}{ }^{27}$ conducted a surveillance on the multisystem inflammatory syndrome in children in the US and found 186 patients in 26 States. Inclusion criteria were serious illness leading to hospitalization, age of less than 21 years, fever that lasted for at least 24 hours, laboratory evidence of inflammation, multisystem organ involvement, and evidence of infection caused by SARS-CoV-2. In this group, the median age was 8.3 years and $62 \%$ of patients were male. Organ-system involvement included the gastrointestinal system (92\%), cardiovascular (80\%), hematological (76\%), mucocutaneous (74\%) and respiratory (70\%). The median duration of hospitalization was seven days. Due to the severity of illness, most children needed intensive care (80\%), mechanical ventilation $(20 \%)$ and vasoactive drugs support (48\%). Four children (2\%) died. Coronary-artery aneurysms were documented in 15 patients $(8 \%)$, and Kawasaki disease-like features were documented in $74(40 \%)$. Most patients (171 or 92\%) had elevations in at least four biomarkers of inflammation.

Elizabeth Dufort, the New York State and the Centers for Disease Control and Prevention formed the Multisystem Inflammatory Syndrome in Children Investigation Team (2020). They identified 191 cases in hospitalized patients younger than 21 years of age, reported by hospitals in the New York State with the diagnosis of Kawasaki disease, toxic shock syndrome, myocarditis, and suspected multisystem inflammatory syndrome associated with COVID-19 in children (MIS-C). In this group of patients, 95 had laboratory-confirmed acute or recent SARS-CoV-2 infection and four patients met clinical and epidemiologic criteria for MIS-C. Thirty-one patients (31\%) were between 0-5 years of old, 42 (42\%) were 6-12 years; 26 (26\%) were 13-20 years. Fifty-three patients (54\%) were male. All of them presented with subjective fever or chills; $97 \%$ had tachycardia, $80 \%$ had gastrointestinal symptoms, $60 \%$ had 
cutaneous rash, 56\% had conjunctivitis and $27 \%$ had mucosal changes. Increased levels of C-Reactive Protein, D-dimer and troponin were found in $100 \%, 91 \%$, and $71 \%$ of the patients, respectively; $62 \%$ received vasopressor drugs, $53 \%$ had evidence of myocarditis, $80 \%$ were admitted to ICUs and two patients died ${ }^{28}$.

\section{COVID-19 in the pediatric population with comorbidities}

Another relevant issue is whether the severity and prognosis of COVID-19 in children and adolescents with comorbidities are worse than in previously healthy counterparts.

Hrusak et al..$^{29}$ investigated 200 children undergoing cancer treatment and nine of them $(4.5 \%)$ contracted COVID-19, eight with mild symptoms and one asymptomatic, suggesting that the coexistence of cancer and COVID-19 did not cause complications.

Minotti et al..$^{30}$ in a systematic review of 16 articles in which 116 adult patients and children with cancer, organ transplantation, primary or secondary immunodeficiencies were analyzed, concluded that immunosuppressed patients had more severe COVID-19, but no worse prognosis, as there were no deaths among the studied patients.

Opposing the previous studies, Shekerdemian et al. ${ }^{31}$ analyzed 48 children and adolescents hospitalized due to COVID-19 in ICUs in Canada and the US. Fifty-two percent were boys with a median age of 13 years, $83 \%$ with comorbidities, $76 \%$ had respiratory symptoms, $38 \%$ required ventilation mechanical, $23 \%$ presented with failure of two or more organs, $4 \%$ died and $31 \%$ remained hospitalized by the time the study was completed. The authors concluded that pediatric SARS-CoV-2 infections are actually less frequent in children than in adults and have a much better prognosis. However, the presence of comorbidities in the pediatric age group seems to act as a risk factor, as in adults, considering the number of symptomatic children with comorbidities who required mechanical ventilation, had multiple organs failure and died.

\section{Maternal, congenital and neonatal infections}

Regarding COVID-19 during pregnancy and the possibility of vertical transmission, data in the literature are still scarce, but suggest that vertical transmission of SARS-CoV-2 may occur, although fetal malformations have not yet been described. Nevertheless, there is evidence that indicates a higher frequency of hospitalizations in pregnant women, as in the context of influenza virus infections, and data around the world indicate a favorable prognosis of COVID-19 in this group. On the contrary, a recent Brazilian review based on the Ministry of Health reported data from February 26 to June 18, 2020, found 124 deaths of pregnant or postpartum women with COVID19, corresponding to a high mortality rate of $12.7 \%$.

Lokken et al..$^{33}$ analyzed 46 pregnant women in the US with a mean age of 29 years, who contracted COVID-19 predominantly in the second and third trimesters, two thirds presenting overweight or obesity. The infection was symptomatic in $93.5 \%$ of women with fever, cough, loss of smell and taste (30\%) and dyspnea. The median duration of symptoms was 24 days. Seven women (15\%) needed hospitalization and one was admitted to the ICU, with a favorable outcome. Severe cases were associated with overweight and obesity, in addition to asthma, diabetes, hypertension and hypothyroidism. There was one induced preterm birth (33 weeks) due to the mother's respiratory failure and the neonate was uninfected. In addition, there was one stillbirth with placenta and fetal tissues testing negative for SARS-CoV-2 and CMV.

In another US study, Pierce-Williams et al. ${ }^{34}$ analyzed a cohort of 64 pregnant women hospitalized due to COVID-19, 69\% with severe infections and $31 \%$ in critical conditions. Comorbidities were pre-existing lung (25\%) and heart disease (17\%), and a mean body mass index of $34 \mathrm{~kg} / \mathrm{m}^{2}$. The mean gestational age was 30 weeks and hospitalizations occurred within seven days after the onset of symptoms and lasted for six days. There was one reversed cardiac arrest and no deaths; $88 \%$ of pregnant women had premature deliveries, $94 \%$ via cesarean sections. There were no stillbirths, neonatal deaths, or vertical transmission of SARS-CoV-2.

Zaigham and Andersson ${ }^{35}$ in a review of 18 articles on pregnant women and neonates published between December 8, 2019 and April 10, 2020, analyzed 108 pregnant women, mostly in the third trimester, with fever $(68 \%)$, cough (34\%), lymphopenia (58\%) and high C-Reactive Protein (70\%). Most women $(91 \%)$ had cesarean sections and three required ICU; there were no maternal deaths, but one intrauterine death and one neonatal death were reported. It was not possible to exclude the vertical transmission of SARS-CoV-2 as fetal and neonatal samples, as well as placentas were not tested.

In Italy, Ferrazzi et al..$^{36}$ studied 42 pregnant women with COVID-19; 24 (57.1\%) had vaginal delivery and 18 (42.9\%) underwent elective cesarean sections. Pneumonia was diagnosed in 19 pregnant women (54.2\%), seven of them $(36.8 \%)$ required respiratory support and four $(21.1 \%)$ needed intensive care. Two women breastfed their newborns without masks because maternal SARS-CoV-2 infection was only suspected after delivery, being confirmed in these two women. The two neonates have also tested positive 
some days after their mothers' diagnoses. In another case, the neonate tested positive a few days after a vaginal delivery, suggesting that this type of delivery may increase the risk of SARS-CoV-2 transmission.

The only case in the literature suggestive of congenital SARS-CoV-2 infection so far, was that of a 29 -year-old Chinese primiparous woman, with 34 weeks and five days of gestational age, presenting with high fever, cough, progressing to respiratory distress. The chest $\mathrm{CT}$ showed a pattern suggestive of COVID-19 and RT-PCR was positive for SARS-CoV-2 in nasopharyngeal secretions. At 35 weeks of gestation, she was hospitalized due to the unfavorable progression of respiratory symptoms, and other four RT-PCRs were performed and remained positive. Three weeks after admission, she underwent serology for SARS-CoV-2, which was also positive (IgM 279.72 AU/mL and $\mathrm{IgG} 107.89 \mathrm{AU} / \mathrm{mL}$ ). On that same occasion, the vaginal secretion was negative for SARS-CoV-2 by RT-PCR. Due to the respiratory conditions, the patient, wearing a N95 mask, underwent a cesarean section and had no contact with the neonate, a girl weighing 3,120 g, with Apgar score of 9 and 10 on the first and fifth minutes of life, without respiratory symptoms. At two hours of life, the neonate's serology was collected and revealed the presence of anti-SARS-CoV-2 $\operatorname{IgG}(140.32 \mathrm{AU} / \mathrm{mL})$ and $\operatorname{IgM}(45.83 \mathrm{AU} / \mathrm{mL})$. Cytokines (IL6 and IL10) were also elevated, as well as the leukocyte count. The chest CT was normal and five RT-PCRs in the neonate's respiratory secretions performed from two hours of life until 160 days of age were negative. Serology was repeated two weeks after the first collection, still revealing high levels of $\operatorname{IgG}(69.94 \mathrm{AU} / \mathrm{mL})$ and detectable, although lower, IgM antibody titers (11.75 AU/mL). Unfortunately, the amniotic fluid and the placenta were not tested for SARS-CoV-2, but the finding of IgM antibodies in the neonate at two hours of life suggests that the infection was transmitted during pregnancy, since IgM antibodies do not cross the placenta as do IgG antibodies ${ }^{37}$.

\section{Diagnosis of COVID-19 by imaging and non-specific laboratory tests}

The chest tomography has been a key exam in elucidating the pulmonary involvement in SARS-CoV-2 infections due to the characteristic images of ground glass patching opacities found until the fourth day after the onset of symptoms, progressing to consolidations in large extensions of the lungs ${ }^{38}$.

Abnormalities in ancillary laboratory exams are also less frequent in children than in adults: blood count with leukopenia, lymphopenia ( $70 \%$ of adults vs. $3.5 \%$ in children), CD4 and CD8 lymphocyte depletion, thrombocytopenia (16-32\%), changes in the neutrophil/ lymphocyte ratio in poorer prognosis cases. Increased lactate dehydrogenase (DHL), aspartate aminotransferase, alanine aminotransferase, C-Reactive Protein (CRP), ferritin, creatine kinase (CK) and D-dimer can also be present, as well as coagulation disorders, thrombotic events and the anti-phospholipid syndrome. Significant increments of IL2, IL4, IL6, IL7, IL10, TNF alpha, Chemokine Ligand 2 (CCL2), CCL3, CCL5, interferon gamma-induced protein 10 (IP10), characterizing the cytokine storm, can be found in more severe COVID-19 cases $^{39}$.

\section{Molecular diagnosis of COVID-19}

The laboratory diagnosis of COVID-19 are based on the detection of viral RNA by real time amplifications $(\mathrm{RT}-\mathrm{PCR})^{40}$ or the detection of antibodies (immunoglobulins) anti-SARS-CoV-2 from the classes IgM, IgA and IgG, produced by the host's immune system. Antibodies can be detected by enzyme immunoassays (ELISA, EIA) whose results are based on optical densities, reflecting the amount of antigen-antibody complexes, or through the detection of chemiluminescent signals, which are proportional to the amount of antigen-antibody complexes present in the sample ${ }^{40-42}$.

After the exposure to the virus, the individual undergoes an incubation period of 1-2 weeks. Viral detection by RT-PCR is possible from one week before the onset of symptoms, reaching a maximum positivity on the week of onset of symptoms. The positivity is still possible during the second week and it rapidly falls onwards ${ }^{41}$.

The molecular assay, called RT-PCR (Reverse Transcriptase-Polymerase Chain Reaction) is a test in which respiratory secretions undergo RNA extraction, followed by a reverse transcription step, in which viral RNA is converted into complementary DNA (cDNA) with the aid of an enzyme, the reverse transcriptase. Then, there is the amplification step in which one or more SARS-CoV-2 targets (fragments of SARS-CoV-2 genes such as the envelope gene (E), nucleocapsid (N), spike (S), RNA-dependent RNA polymerase (RdRP), several ORFs, are amplified and these amplification products are identified and hybridized by fluorochrome-labeled probes, making amplification more specific, but at a higher cost. Although it is possible to quantify the number of viral copies of the target genes, in most studies RT-PCR for SARS-CoV-2 has been used only qualitatively, generally releasing positive or negative results, which should be understood by clinicians as viruses detected or not detected in that specific sample ${ }^{40}$.

Hase et al..$^{43}$ reported a case of COVID-19 with pneumonia and negative RT-PCR in an oropharyngeal 
swab. New oropharyngeal and induced sputum specimens were collected five days after hospitalization, being positive in sputum and negative in oropharynx, indicating that a negative RT-PCR in respiratory samples is not enough to rule out COVID-19. In addition, three patients presenting with chest computed tomography suggestive of COVID-19 but persistent negative RT-PCR from days 6 to 8 after the onset of symptoms, were very likely to be false-negatives of RT-PCR, suggesting a lack of sensitivity of RT-PCR in nasopharyngeal swabs depending on the time elapsed from the onset of symptoms ${ }^{14}$.

Even with sensitivity limitations, the detection of viral RNA is considered the gold standard for the diagnosis of COVID-19 in respiratory secretions, however, false negative results in the order of $25-30 \%$ have been reported. The detection of the virus depends on several aspect: presence of sufficient viruses in the sample, collection of respiratory samples during the "ideal" phase for viral detection, ideally within the first week after the onset of symptoms, adequate collection protocols, samples storage and processing procedures. Inadequate procedures can limit the performance of molecular tests. RT-PCR sensitivity also varies according to the type of biological material. In a study of 1,070 samples from 250 patients with COVID-19, the sensitivity of RT-PCR performed with ORF1ab primers was: bronchoalveolar lavage (93\%), sputum (72\%), nasal swab (63\%), oropharynx swab (32\%), feces (29\%), blood (1\%) and there was no detection in urine samples. Respiratory secretion swabs were collected 1-3 days after the onset of symptoms. Although the best biological material would the bronchoalveolar lavage (93\% sensitivity), in clinical practice this invasive procedure is not feasible and for this reason the analysis of nasopharyngeal and oropharyngeal (63\%) and oropharynx (32\%) secretions combined was adopted, expecting the analysis to reach around $70 \%$ sensitivity ${ }^{44}$.

Although there is no consensus, the most widely used RT-PCRs in the world to detect SARS-CoV-2 are those recommended by the Centers for Disease Control (CDC) of USA ${ }^{45}$, the Charité Virology (Berlin, Germany) ${ }^{46}$, and the Department of Virology at the University of Hong Kong Medical School and School of Public Health ${ }^{47}$.

In the RT-PCR recommended by the CDC-USA, there is a simultaneous amplification of three distinct regions of the viral nucleocapsid $(\mathrm{N} 1, \mathrm{~N} 2$ and $\mathrm{N} 3)$ and of a control region from the RNAse $\mathrm{P}$, with hybridization of amplification products by fluorescent probes. The test is positive when it amplifies N1, N2 and N3 in addition to RNAse $\mathrm{P}$, and is negative when only RNAse $\mathrm{P}$ is amplified. The test is invalid when none of the amplification products appears and is inconclusive (must be repeated after a new
RNA extraction) if the RNAse $\mathrm{P}$ is positive together with $\mathrm{N} 1$ or $\mathrm{N} 2$ or $\mathrm{N} 3^{45}$.

In the diagnostic system described by Corman et al. ${ }^{46}$ in Germany (Charité Virology), there is a first RT-PCR performed with primers from the envelope gene (E) that detects the subgenus Sarbecovirus of the B-line of coronaviruses. These primers will recognize both, SARS$\mathrm{CoV}$ and SARS-CoV-2, and amplification products are detected by a fluorescent probe. In the second RT-PCR, the target gene is RdRP, and in addition to the primer pair, there are two probes labeled with distinct fluorochromes, one recognizing SARS-CoV and SARS-CoV-2 and one specific to SARS-CoV-2.

In the RT-PCR proposed by the Hong Kong University (HKU), two reactions are carried out separately, the first one with ORF1b primers (nsp14) and the second one targeting the $\mathrm{N}$ gene (viral nucleocapsid). The two amplification products are recognized and hybridized to fluorescent probes ${ }^{47}$.

Initial studies indicated that the RT-PCR performed with primers from the RdRP and E genes had high analytical sensitivity, being able to detect 3.6 - 3.9 viral copies per reaction, while the amplifications performed with primers from the nucleocapsid gene detect 8.3 viral copies per reaction $^{48}$. However, commercial RT-PCR kits for the detection of SARS-CoV-2 targeting the E, N and ORF $1 \mathrm{ab}$ genes did not perform well when clinical samples were tested, reaching sensitivities ranging from 968 copies $/ \mathrm{mL}$ to 7,744 copies/mL, making the occurrence of false negatives very likely ${ }^{49}$.

\section{Serological diagnosis of COVID-19 (Anti-SARS-CoV-2 antigen or antibody detection)}

Serological assays, such as rapid immunochromatographic tests or immune assays with simple reading of optical densities or detection of fluorescent or chemiluminescent signals are faster, less expensive and easier to perform than RT-PCR $^{40-42}$.

Anti-SARS-CoV-2 IgM antibodies can be detected in COVID-19 between 3-7 days after the onset of symptoms, on week 2 of illness, reaching peak levels on week 3 and beginning to decrease on weeks 4 and 5, but they can still be detected, depending on the method, on week 6 . Regarding the production of IgA antibodies, some studies have reported their appearance at the same time as IgM antibodies and others, along with $\operatorname{IgG}$ antibodies. In any case, in COVID-19, IgA antibodies seem to present with higher levels than IgM, with some studies indicating a more adequate diagnostic performance of the combined analysis of $\mathrm{IgA}$ and $\mathrm{IgG}$ antibodies in relation to IgM and IgG. As for IgG antibodies, some studies have detected IgA antibodies 
a few days after IgM antibodies, while others, beginning 14 days after the onset of symptoms, remaining detectable for at least 6 weeks, but there is no information on long-term protection provided by these antibodies ${ }^{40,41}$.

Lassaunière et al. ${ }^{42}$ compared the efficiency of nine commercial tests, three ELISA and six immunochromatographic kits. Specificities were $100 \%$ for Wantai SARS-CoV-2 total antibody ELISA kit, 93\% for Euroimmun IgA ELISA and $96 \%$ for Euroimmun IgG ELISA with sensitivities of $90 \%, 90 \%$ and $65 \%$, respectively. Among the six immunochromatographic kits (Dynamiker Biotechnology, CTK Biotech, AutoBio Diagnostics and Artron Laboratories) only Acro Biotech (80\%) and Hangzhou All Test Biotech (87\%) showed specificity problems in detecting IgM. Then, sensitivities were analyzed in four periods: up to 6 days of infection, 7-13 days, 14-20 days and $>21$ days. For ELISA kits, sensitivities ranged from $40-86 \%$ in the earliest phase, $67-100 \%$ in the intermediate phase and $78-89 \%$ in the last two phases, with the Chinese kit performing better than those from Euroimmun. The performance of the immunochromatographic kits was worse than the ELISAs in the early phase, but it was comparable to ELISA Wantai Total Ab ELISA and ELISA Euroimmun IgA considering the four periods combined.

Huang et al..$^{50}$ evaluated an ELISA assay for SARS-CoV-2 in plasma samples of confirmed cases of COVID-19 or suspected cases with negative RT-PCR. In those with confirmed diagnosis, the average duration of $\operatorname{IgM}$ and $\operatorname{IgA}$ antibodies in the circulation was five days, and $\mathrm{IgG}$ antibodies were detected from the $14^{\text {th }}$ day after the onset of symptoms. ELISA-IgM was more sensitive than RT-PCR in detecting cases from the $5^{\text {th }}$ day after the onset of symptoms.

In the case of SARS-CoV-2, the main target of neutralizing antibodies is precisely the $S$ protein, more specifically the RBD (receptor binding domain) region that will inhibit the viral recognition by the ACE2 cell receptor. Wu et al. ${ }^{51}$, followed-up the neutralizing antibody levels in 175 adult patients with mild or moderate COVID-19 by means of a sensitive and reproducible neutralization assay. The mean age of the patients was 50 years, with $53 \%$ of women. The average hospital stay was 16 days, with the disease lasting 21 days. Antibodies detection used a neutralization assay with a pseudovirus (PsV), a technique widely used for the quantification of neutralizing antibodies in other viral infections, as the ones caused by influenza viruses, Ebola virus, MERS$\mathrm{CoV}$ and SARS-CoV. The quantification of neutralizing antibodies from recovered individuals was determined on the day of hospital discharge. Low levels of neutralizing antibodies were detected until the $10^{\text {th }}$ day. From that day until the $150^{\text {th }}$ day of infection, concentrations of neutralizing antibodies have raised and remained stable afterwards, however, only $14 \%$ of the patients had high titers of neutralizing antibodies, in general older patients who had more prolonged infections, being only these the potential donors of plasma for patients with COVID-19 in severe or critical conditions.

The immunochromatographic tests with the best performance to date are the Colloidal-GoldImmunochromatographic Assays (GICA). Xiang et al. ${ }^{52}$ tested 126 serum samples from COVID-19 patients, with $57.1 \%$ IgM sensitivity, $100 \%$ specificity and $69 \%$ accuracy. For IgG, they found $81.3 \%$ sensitivity, $100 \%$ specificity and $86.5 \%$ accuracy. The combined sensitivity (IgM and IgG) was $82.4 \%$.

Pan et al..$^{53}$ also using GICA, tested 134 samples from patients with COVID-19 confirmed by RT-PCR, in addition to suspected cases with negative RT-PCR. The combined sensitivity (IgM and $\operatorname{IgG}$ ) was $11.1 \%$ at the beginning of the infection (1-7 days after the onset of symptoms), 92.9\% between 8-14 days and $96.8 \%$ after 15 days. Surprisingly, the detection of $\operatorname{IgG}$ in suspected cases with negative RT-PCR was $43.6 \%$.

\section{Management of pediatric patients with COVID-19}

Knowledge on the treatment of SARS-CoV-2 is rapidly evolving, and it is essential that pediatricians are up to date, looking systematically for new guidelines and recommendations on the management of COVID-19 in childhood $^{54}$.

There is no definitive evidence of any specific effective therapy for treating children with COVID-19 so far. The outpatient management of children consists basically of symptomatic and supportive care, along with isolation measures. Children at home should be closely monitored for signs of clinical deterioration: shortness of breathe or severe respiratory distress, chest pain, cyanosis and clinical signs associated with shock. Clinical deterioration may occur suddenly and immediate reevaluation by a pediatrician is recommended ${ }^{54,55}$.

The length of home isolation is uncertain and depends on the duration of viral shedding which in turn is also variable. The WHO criteria (WHO 2020) for discharging patients from isolation without requiring a new specific test to SARS-CoV-2 are 10 days after the onset of symptoms for symptomatic patients, in addition to at least three additional days without symptoms. In the case of asymptomatic patients, at least 10 days after the last positive test to SARS-CoV-2 ${ }^{56}$. 
Children with severe clinical manifestations should be hospitalized. The basis for the management of COVID-19 infections in hospitalized children is to provide supportive treatment ${ }^{55,56}$. The team responsible for the care of the child must ensure an adequate respiratory support, including supplemental oxygen and noninvasive or invasive ventilatory support, as needed. Children with severe illness and respiratory failure need special attention regarding the adequate fluid and electrolyte supplementation and they should be monitored for bacterial pneumonia and other secondary infections, with strict clinical control and through cultures and other microbiological tests. The empirical use of antibiotics may be initially necessary, but their maintenance should be based, whenever possible, on the identification of bacteria by culture method $\mathrm{s}^{57}$.

There is a paucity of data on the use of antivirals for COVID19 treatment in the pediatric population, and there is no indisputable evidence in the medical literature to support their use in children to date. Antivirals may be considered only for children with severe or critical disease in the presence of a positive test to SARS-CoV-2 and on a case-by-case basis. As there are no definitive data establishing what are the risk factors to be considered in criteria for the progression of COVID-19 to a serious or critical medical condition in children, a panel of experts extrapolated data from adult patients to establish possible risk factors in children that could be used to indicate the prescription of antivirals, such as the presence of immunological impairment, especially the ones revealing a severe immunocompromised status, young age, severe underlying cardiac and/or pulmonary disease, obesity, diabetes, among other conditions ${ }^{57}$.

As there are no definitive studies on the use of antivirals for COVID-19 in children, the panel of experts recommended that their use should be restricted only to severe cases, when potential benefits not yet perceived could outweigh possible risks and injuries, and whenever possible within a clinical trial. If an antiviral is to be used, remdesivir is, for the moment, the drug of choice ${ }^{57}$.

For children presenting with the multisystem inflammatory syndrome (MIS-C), there are currently no published guidelines from WHO, CDC or ECDC regarding the treatment of COVID-19, or studies comparing the efficacy of various treatment options. A group of researchers from the University of California made some recommendations for the management of MIS-C based on the extrapolation of data from other syndromes. Children with clinical features meeting the criteria for Kawasaki disease should be treated according to current recommendations, with high doses of intravenous immunoglobulins and aspirin. For children with severe disease or persistent inflammation, corticosteroids should be considered. In children with clinical manifestations consistent with a secondary hemophagocytic lymphohistiocytosis activation syndrome, the initial use of anakinra (recombinant and slightly modified version of the human interleukin 1 receptor antagonist protein) and intravenous immunoglobulins is suggested. The addition of pulsed corticosteroids and other immunosuppressive agents, such as cyclosporine and tacrolimus can be considered in severe cases ${ }^{58}$.

\section{CONCLUSION}

Although the advances made in the research of SARS-CoV-2 and COVID-19 in such a short time are undeniable, at present we still do not have a consensual etiological treatment or a vaccine with proven efficacy. Even so, it is important that pediatricians are kept up to date in relation to the particularities of COVID-19 in pediatric patients, new findings on the virus itself, its characteristics and diagnosis and new therapeutic approaches.

\section{AUTHORS' CONTRIBUTIONS}

LY: made the final selection of the most interesting articles that would be part of the review based on the summary on molecular biology, serology, virology, epidemiology, and physiopathology. Made the selection of the articles on maternal, fetal and neonatal infections; EHS: carried out a survey of the bibliography on the molecular diagnosis and summarized the selected articles; LSP: carried out the survey of the bibliography on the serological diagnosis and summarized the selected articles; MCR: carried out the survey of the bibliography on the virological and epidemiological aspects of SARS-CoV-2, SARS-CoV and MERS and summarized the selected articles; KAK: carried out the survey of the bibliography the physiopathology of SARS-CoV-2 infections, summarized the selected articles and organized all the references of the manuscript; MGV: revised the manuscript as a specialist in Pediatric Infectious Diseases and wrote the management of patients' section; TSO: idealized the review article, selected the manuscripts of children with and without comorbidities and COVID19 and wrote the final manuscript.

\section{CONFLICT OF INTERESTS}

All the authors have read and approved the final version of the manuscript and declare that they have no conflict of interests whatsoever. 


\section{REFERENCES}

1. World Health Organization. Criteria for releasing COVID-19 patients from isolation. [cited $2020 \mathrm{Aug} 21$ ]. Available from: https://www.who.int/publications/i/item/criteria-for-releasingcovid-19-patients-from-isolation

2. de Wit E, van Doremalen N, Falzarano D, Munster VJ. SARS and MERS: recent insights into emerging coronaviruses. Nat Rev Microbiol. 2016;14:523-34.

3. Kasibhatla SM, Kinikar M, Limaye S, Kale MM, Kulkarni-Kale U. Understanding evolution of SARS-CoV-2: a perspective from analysis of genetic diversity of RdRp gene. J Med Virol. 2020 In Press.

4. Sheikh JA, Singh J, Singh H, Jamal S, Khubaib M, Kohli S, et al. Emerging genetic diversity among clinical isolates of SARSCoV-2: lessons for today. Infect Genet Evol. 2020;84:104330.

5. Rothan HA, Byrareddy SN. The epidemiology and pathogenesis of coronavirus disease (COVID-19) outbreak. J Autoimmun. 2020;109:102433.

6. Baud D, Qi X, Nielsen-Saines K, Musso D, Pomar L, Favre G. Real estimates of mortality following COVID-19 infection. Lancet Infect Dis. 2020;20:773.

7. Pan Y, Yu X, Du X, Li Q, Li X, Qin T, et al. Epidemiological and clinical characteristics of 26 asymptomatic SARS-CoV-2 carriers. J Infect Dis. 2020;221:1940-7.

8. Gheblawi M, Wang K, Viveiros A, Nguyen Q, Zhong JC, Turner $\mathrm{AJ}$, et al. Angiotensin-converting enzyme 2: SARS-CoV-2 receptor and regulator of the Renin-Angiotensin system: celebrating the 20th anniversary of the discovery of ACE2. Circ Res. 2020;126:1456-74.

9. Castagnoli R, Votto M, Licari A, Brambilla I, Bruno R, Perlin S, et al. Severe acute respiratory syndrome Coronavirus 2 (SARSCoV-2) infection in children and adolescents: a systematic review. JAMA Pediatr. 2020 In Press.

10. Guo YR, Cao QD, Hong ZS, Tan YY, Chen SD, Jin HJ, et al. The origin, transmission and clinical therapies on coronavirus disease 2019 (COVID-19) outbreak - an update on the status. Mil Med Res. 2020;7:11.

11. Cheung KS, Hung IF, Chan PP, Lung KC, Tso E, Liu R, et al. Gastrointestinal manifestations of SARS-CoV-2 infection and virus load in fecal samples from the Hong Kong cohort and systematic review and meta-analysis. Gastroenterology. 2020;159:81-95.

12. Ronco C, Reis T. Kidney involvement in COVID-19 and rationale for extracorporeal therapies. Nat Rev Nephrol 2020;16:308-10.

13. Mao L, Jin H, Wang M, Hu Y, Chen S, He Q, et al. Neurologic manifestations of hospitalized patients with coronavirus disease 2019 in Wuhan, China. JAMA Neurol. 2020 In Press.

14. Xu J, Wu R, Huang H, Zheng W, Ren X, Wu N, et al. Computed tomographic imaging of 3 patients with Coronavirus disease 2019 pneumonia with negative virus real-time reverse- transcription Polymerase Chain Reaction test. Clin Infect Dis. 2020;71:850-2.

15. Bunyavanich S, Do A, Vicenzio A. Nasal gene expression of Angiotensin-converting enzyme 2 in children and adults. JAMA. 2020;323:2427-9.

16. Chen L, Liu M, Zhang Z, Qiao K, Huang T, Chen M, et al. Ocular manifestations of a hospitalised patient with confirmed 2019 novel coronavirus disease. Br J Ophthalmol. 2020;104:74851.

17. Colonna C, Monzani NA, Rocchi A, Gianotti R, Boggio F, Gelmetti C. Chilblain-like lesions in children following suspected COVID-19 infection. Pediatr Dermatol. 2020;37:437-40.

18. Ghazizadeh Z, Majd H, Richter M, Samuel R, Zekavat SM, Asgharian $\mathrm{H}$, et al. Androgen regulates SARS-CoV-2 receptor levels and is associated with severe COVID-19 symptoms in men. BioRxiv. 2020 In Press.

19. Li Ya, Zhou W, Yang L, You R. Physiological and pathological regulation of ACE2, the SARS-CoV-2 receptor. Pharmacol Res. 2020;157:104833.

20. Ciaglia E, Vecchione C, Puca AA. COVID-19 infection and circulating ACE2 levels: protective role in women and children. Front Pediatr. 2020;8:206.

21. Zimmermann P, Curtis N. Coronavirus infections in children including COVID-19: an overview of the epidemiology, clinical features, diagnosis, treatment and prevention options in children. Pediatr Infect Dis J. 2020;39:355-68.

22. Ong JS, Tosoni A, Kim YJ, Kissoon N, Murthy S. Coronavirus disease 2019 in critically ill children: a narrative review of the literature. Pediatr Crit Care Med. 2020;21:662-6.

23. Liguoro I, Pilotto C, Bonanni1 M, Ferrari ME, Pusiol A, Nocerino A, et al. SARS-COV-2 infection in children and newborns: a systematic review. Eur J Pediatr. 2020 In Press.

24. Lu X, Zhang L, Du H, Zhang J, Li YY, Qu J, et al. SARS-CoV-2 infection in children. New Engl J Med. 2020;382:1663-5.

25. Götzinger F, Santiago-García B, Noguera-Julián A, Lanaspa M, Lancella L, Carducci FI, et al. COVID-19 in children and adolescents in Europe: a multinational, multicentre cohort study. Lancet Child Adolesc Health. 2020;4:653-61.

26. Jiang S, Liu P, Xiong G, Yang Z, Wang M, Yan Li Y, et al. Coinfection of SARS-CoV-2 and multiple respiratory pathogens in children. Clin Chem Lab Med. 2020;58:1160-1.

27. Feldstein LR, Rose EB, Horwitz SM, Collins JP, Newhams MM, Son MB, et al. Multisystem inflammatory syndrome in U.S. children and adolescents. N Engl J Med. 2020;383:334-46.

28. Dufort EM, Koumans EH, Chow EJ, Rosenthal EM, Muse A, Rowlands $\mathrm{J}$, et al. Multisystem inflammatory syndrome in children in New York State. Engl J Med. 2020;383:347-58.

29. Hrusak O, Kalina T, Wolf J, Balduzzi A, Provenzi M, Rizzari $\mathrm{C}$, et al. Flash survey on severe acute respiratory syndrome Coronavirus- 2 infections in paediatric patients on anticancer treatment. Eur J Cancer. 2020;132:11-6. 
30. Minotti C, Tirelli F, Barbieri E, Giaquinto C, Donà D. How is immunosuppressive status affecting children and adults in SARS-CoV-2 infection? A systematic review. J Infect. 2020;81:e61-6

31. Shekerdemian LS, Mahmood NR, Wolfe KK, Riggs BJ, Ross $\mathrm{CE}, \mathrm{McKiernan} \mathrm{CA}$, et al. Characteristics and outcomes of children with Coronavirus disease 2019 (COVID-19) infection admitted to US and Canadian pediatric intensive care units. JAMA Pediatr. 2020 In Press.

32. Takemoto ML, Menezes MO, Andreucci CB, Nakamura-Pereira M, Amorim MM, Katz L, et al. The tragedy of COVID-19 in Brazil: 124 maternal deaths and counting. Int J Gynecol Obstet. 2020 In Press.

33. Lokken EM, Walker CL, Delaney S, Kachikis A, Kretzer NM, Erickson A, et al. Clinical characteristics of 46 pregnant women with a SARS-CoV-2 infection in Washington State. Am J Obstet Gynecol. 2020 In Press.

34. Pierce-Williams RA, Burd J, Felder L, Khoury R, Bernstein PS, Avila K, et al. Clinical course of severe and critical COVID-19 in hospitalized pregnancies: a US cohort study. Am J Obstet Gynecol MFM. 2020 In Press.

35. Zaigham M, Andersson O. Maternal and perinatal outcomes with COVID-19: a systematic review of 108 pregnancies. Acta Obstet Gynecol Scand. 2020;99:823-9.

36. Ferrazzi E, Frigerio L, Savasi V, Vergani P, Prefumo F, Barresi S, et al. Vaginal delivery in SARS-CoV-2-infected pregnant women in Northern Italy: a retrospective analysis. BJOG. 2020 In Press.

37. Dong L, Tian J, He S, Zhu C, Wang J, Liu C, et al. Possible vertical transmission of SARS-CoV-2 from an infected mother to her newborn. JAMA. 2020;323:1846-8.

38. Pan F, Ye T, Sun P, Gui S, Liang B, Li L, et al. Time course of lung changes on chest CT during recovery from 2019 novel Coronavirus (COVID-19) pneumonia. Radiology. 2020;295:715-21.

39. Henry BM, Benoit SW, Oliveira MH, Hsieh WC, Benoit J, Ballout RA, et al. Laboratory abnormalities in children with mild and severe coronavirus disease 2019 (COVID-19): a pooled analysis and review. Clin Biochem. 2020;81:1-8.

40. Oliveira BA, Oliveira LC, Sabino EC, Okay TS. SARS-CoV-2 and the COVID-19 disease: a mini review on diagnostic methods. Rev Inst Med Trop Sao Paulo. 2020;62:e44.

41. Lee CY, Lin RT, Renia L, Ng LF. Serological approaches for COVID-19: epidemiologic perspective on surveillance and control. Front Immunol. 2020;11:879.

42. Lassaunière R, Frische A, Harboe ZB, Nielsen AC, Fomsgaard A, Krogfelt KA, et al. Evaluation of nine commercial SARSCoV-2 immunoassays. MedRxiv. 2020 In Press.

43. Hase R, Kurita T, Muranaka E, Sasazawa H, Mito H, Yano Y. A case of imported COVID-19 diagnosed by PCR-positive lower respiratory specimen but with PCR-negative throat swabs. Infect Dis (Lond). 2020;52:423-6.
44. Wang W, Xu Y, Gao R, Lu R, Han K, Wu G, et al. Detection of SARS-CoV-2 in different types of clinical specimens. JAMA. 2020;323:1843-4.

45. Centers for Disease Control and Prevention. CDC 2019-novel Coronavirus (2019-nCoV) real-time RT-PCR diagnostic panel. [cited 2020 Aug 21]. Available from: https://www.fda.gov/ media/134922/download

46. Corman VM, Landt O, Kaiser M, Molenkamp R, Meijer A, Chu DK, et al. Detection of 2019 novel coronavirus (2019-nCoV) by real-time RT-PCR. Euro Surveill. 2020;25:2000045.

47. Chu DK, Pan Y, Cheng SM, Hui KP, Krishnan P, Liu Y, et al. Molecular diagnosis of a novel Coronavirus (2019-nCoV) causing an outbreak of pneumonia. Clin Chem. 2020;66:54955.

48. Corman V, Bleicker T, Brünink S, Drosten C, Landt O, Koopmans M, et al. Diagnostic detection of Wuhan Coronavirus 2019 by real-time RT-PCR. [cited 2020 Aug 21]. Available from: https://www.who.int/docs/default-source/coronaviruse/ wuhan-virus-assay-v1991527e5122341d99287a1b17c111902. pdf?sfvrsn=d381fc88_2

49. Li Y, Lin Y, Li J, Chen L, Song Y, Cai Z, et al. Stability issues of RTPCR testing of SARS-CoV-2 for hospitalized patients clinically diagnosed with COVID-19. J Med Virol. 2020;92:903-8.

50. Huang AT, Garcia-Carreras B, Hitchings MD, Yang B, Katzelnick L, Rattigan SM, et al. A systematic review of antibody mediated immunity to coronaviruses: antibody kinetics, correlates of protection, and association of antibody responses with severity of disease. medRxiv. 2020 In Press.

51. Wu F, Wang A, Liu M, Wang Q, Chen J, Xia S, et al. Neutralizing antibody responses to SARS-CoV-2 in a COVID-19 recovered 2 patient cohort and their implications. medRxiv. 2020 In Press.

52. Xiang J, Yan M, Li H, Liu T, Lin C, Huang S, et al. Evaluation of enzyme-linked immunoassay and colloidal goldimmunochromatographic assay kit for detection of novel Coronavirus (SARS-Cov-2) causing an outbreak of pneumonia (COVID-19). medRxiv. 2020 In Press.

53. Pan Y, Li X, Yang G, Fan J, Tang Y, Zhao J, et al. Serological immunochromatographic approach in diagnosis with SARS-CoV-2 infected COVID-19 patients. J Infect. 2020;81:e28-32.

54. National Institutes of Health. Coronavirus disease 2019 (COVID-19) treatment guidelines. [cited 2020 Aug 21]. Available from: https://covid19treatmentguidelines.nih.gov

55. Chiotos K, Hayes M, Kimberlin DW, Jones SB, James SH, Pinninti $\mathrm{SG}$, et al. Multicenter initial guidance on use of antivirals for children with COVID-19/SARS-CoV-2. J Ped Infect Dis Soc 2020 In Press.

56. Bhimraj A, Morgan RL, Shumaker AH, Lavergne V, Baden L, Cheng VC, et al. Infectious Diseases Society of America Guidelines on the Treatment and Management of Patients with COVID-19. Clin Infect Dis. 2020 In Press. 
Yamamoto et al.

57. Rawson TM, Moore LS, Zhu N, Ranganathan N, Skolimowska $\mathrm{K}$, Gilchrist $\mathrm{M}$, et al. Bacterial and fungal co-infection in individuals with coronavirus: a rapid review to support COVID-19 antimicrobial prescribing. Clin Infect Dis. 2020 In Press.
58. Nakra NA, Blumberg DA, Herrera-Guerra A, Lakshminrusimha S. Multi-system inflammatory syndrome in children (MIS-C) following SARS-CoV-2 infection: review of clinical presentation, hypothetical pathogenesis, and proposed management. Children (Basel). 2020;7:69.

\section{ERRATUM}

Page 1, where it reads:

Lacyane Silva Pinto

Should be read:

Lacyane Santos Pinto

http://doi.org/10.1590/S1678-9946202062065err 